\title{
On geometric phase from pure projections
}

\author{
Rajendra Bhandari
}

\author{
Raman Research Institute, \\ Bangalore 560 080, India. \\ email: bhandari@rri.ernet.in
}

submitted to Journal of Modern Optics.

MS version of 4 February 1998 


\begin{abstract}
The relation between three recent experiments aimed at demonstrating a strictly geometric phase using pure projections is clarified in the light of a recent claim to such a measurement. The possibility of observing $\pm 2 \pi$ phase jumps in an optical interference experiment is pointed out.
\end{abstract}




\section{Introduction}

Recently, Hariharan et.al. [1] have reported an experiment (HRSS Expt.) in which they extend the work of Berry and Klein [2] (BK Expt.) to measure a pure geometric phase arising from state projections, as formulated by Pancharatnam [3]. These experiments are claimed [1] to be intrinsically free from all sources of dynamical phase.

In this paper I show that (i) there are difficulties in regarding the HRSS Expt. as a measurement of a pure geometric phase since it is not intrinsically free from dynamical phase as claimed, (ii) an experiment earlier reported in literature [4] (the IWBS Expt.) did measure a pure geometric phase arising from state projections. In addition to measuring a linearly increasing geometric phase, it measured in detail the nonlinear behaviour and jump of the geometric phase through $\pm \pi$ near a singularity and explicitly verified the existence of the singularity associated with the geometric phase, (iii) the BK Expt. and the HRSS Expt. contain similar singularities which could be seen if the techniques used and the available sensitivities allowed a more complete sampling of the parameter spaces in these experiments and (iv) in the HRSS expt. one could, in principle, see phase jumps equal to $\pm 2 \pi$, a somewhat surprising result.

\section{Historical remarks}

Following Berry's discovery of the adiabatic phase [5], there was a widespread revival of interest in Pancharatnam's work on polarization of light [6], in particular, in the form of experimental demonstrations of the geometric phase by interferometry, the first one being an experiment by Bhandari and Samuel [7]. This was followed by a number of experiments by other groups [8, 9, 10, 11, 12. 
A common feature of all these experiments was that these involved circuits on the Poincaré sphere consisting of geodesic curves, caused by unitary transformations. For a unitary transformation which takes the state along a geodesic, the dynamical phase is zero [13], as in case of circuits caused by projections, considered by Pancharatnam [3]. The geodesic nature of the circuits is ensured in these experiments by appropriate choice of the polarization elements like retarders and their orientation. Departures from a geodesic circuit would arise in these experiments only from errors in the retardation and orientations. The other common feature of these experiments was that these measured a geometric phase increasing linearly as a function of an experimental parameter, typically rotation angle of an optical element. A more recent measurement of such a linear phase using neutron interferometry has been reported by Wagh et.al. [14].

Hariharan and Roy reported a new version of the above experiments in which they use a Sagnac interferometer configuration [15]. Since the two interfering beams in this experiment travel the same physical path in space, all $\mathrm{U}(1)$ phase errors (errors in the polarization-independent part of the phase) are eliminated in this experiment. However since the beams still encounter a beam splitter and mirrors this experiment is not free from $\mathrm{SU}(2)$ phase errors caused by unknown polarization changes brought about by these optical elements. Moreover this experiment does not measure fringe shifts. It measures the modulation of intensity of the superposed beams as a function of orientation of a halfwave plate which is interpreted as a linearly increasing difference of the geometric phase shifts acquired by the clockwise and the counterclockwise beams.

Following this, the present author reported an experiment [4] (the IWBS 
Expt.) that directly measures phase shifts in which the two interfering beams, with orthogonal polarizations, are not split, hence they do not encounter any beam splitter or mirrors 1 . This eliminates the $\mathrm{SU}(2)$ errors as well. Yet the two beams acquire a geometric phase difference. This idea was incorporated by Hariharan and Roy in their new technique for phase stepping interferometry [16, 17].

\section{The IWBS Experiment}

This experiment [4] is performed with a Hewlett Packard laser interferometer that uses heterodyne techniques to measure and record phase shifts between two light waves at He-Ne laser frequency with a sensitivity of $\lambda / 20$, alongwith its sign. It uses a novel interferometer configuration in which the two interfering beams with orthogonal polarizations, represented by the points $A$ and $\tilde{A}$ on the Poincaré sphere (figure 1), travel along the same path in space, pass through a quarterwave plate so oriented as to take the two beams to a chosen pair of orthogonal polarization states represented by the points $P$ and $\tilde{P}$ and then pass through a linear polarizer which passes the state represented by $\mathrm{N}$ (figure 1). The phase difference between the beams is continuously recorded as a function of rotation of the polarizer resulting in rotation of the point $\mathrm{N}$ on the sphere. The experiment is performed for several orientations of the quarterwave plate $\mathrm{P}$ which determine the polar angles $\theta$ and $180^{\circ}-\theta$ of the points $P$ and $\tilde{P}$. The phase shift measured in this experiment as the polarizer is rotated from $\mathrm{Q}$ to $\mathrm{N}$ is given by the phase of the complex number $<P|Q><Q| \tilde{P}><\tilde{P} \mid$ $N><N \mid P>$, where $|P>| N>,,|\tilde{P}>| Q>$, are respectively the two-

\footnotetext{
${ }^{1}$ The label IWBS stands for "Interferometry Without Beam Splitters"
} 
component spinors representing the states $P, N, \tilde{P}, Q$ and is equal to half the solid angle subtended by the shaded area bounded by the two geodesics $P N F \tilde{P}$ and $\tilde{P} L Q P$. It is thus a pure geometric phase arising from four projections.

Figure 2 reproduces from ref. 四 the expected phase shift (solid line) and the measured values (dots) as a function of the orientation of the linear polarizer for several different values of $\theta$. For $\theta=0^{\circ}$ and $\theta=180^{\circ}$, the phase shift is linear and corresponds to the situation studied in the HRSS expt. For other values of $\theta$, the phase shift is nonlinear and for values close to $\theta=90^{\circ}$, the curves are highly nonlinear near critical orientations of the polarizer, switching sign at $\theta=90^{\circ}$. The singular behaviour occurs when the state $|N\rangle$ becomes orthogonal to the state $\mid P>$ or $\mid \tilde{P}>$ and one of the scalar products in the above product becomes zero. The jump in the phase near the singularity in these experiments is equal to $\pm \pi$. Such jumps were first predicted in ref. [18] and their relevance to the phase evolution of quantum symstems pointed out in refs. [18, 19].

Another independent observation of the nonlinearity of the Pancharatnam phase was reported by Schmitzer et. al. [20]. This experiment, however, does not detect the switch in the sign of the phase and the singularity.

\section{The HRSS Experiment}

In this experiment [1], the authors use a Sagnac interferometer configuration with a nonpolarizing beam splitter and three mirrors and a rotatable linear analyser LA placed between two of the mirrors. A He-Ne laser beam rendered linearly polarized by means of a linear polarizer LP (fig. 1 of ref. [1]) is then converted into left circular by means of a quarterwave plate before incidence on 
the beam splitter where it is divided into a clockwise and a counterclockwise beam. The beams then pass through a linear polarizer LA in the opposite directions and recombine at the beam splitter. The two beams then pass through a common right circular analyzer and their combined intensity sensed with a photodiode. The variation of this intensity at a given point in space is monitored as a function of the orientation of LA, i.e. $\phi / 2$ ( or $\theta$ in the notation of ref.1). From an observed sinusoidal variation of the intensity, the authors infer a change in geometric phase linear in $\phi$.

Such an interpretation of the observed intensity curve has several problems. First of all it is not equivalent to measurement of a phase shift as a function of $\phi$. This would be so if for example a spatial shift of interference fringes on a screen were measured directly. Alternatively, phase shifts could be measured by heterodyne interferometry where these could be looked upon as fringe shifts in the time domain. Secondly, it is not true that the beams do not undergo any $\mathrm{SU}(2)$ transformation during the passage through the interferometer. Every reflection from a mirror or a beam splitter introduces an $\mathrm{SU}(2)$ transformation on the polarization state of the beam. For reflection off an ideal metal mirror, this could be taken to be equivalent to that due to a halfwave plate [21]. In practice the reflections are not ideal. Beam splitters in particular are notorious for introducing undesirable polarization changes. As the linear analyzer LA is rotated, a variable polarization is incident on the beam splitter and an $\mathrm{SU}(2)$ transformation varying with $\phi$ would be introduced. The evolution due to pure projections is therefore accompanied by $\mathrm{SU}(2)$ evolution. This introduces a dyamical phase error which is a function of the orientation of the polarizer, hence significant. Since fringe shifts are not measured, the phase errors due to these effects cannot be easily estimated. 
Finally, we show that if the experiment were performed in the fringe-shift mode and adequate sensitivity available, one could see a singularity in this experiment too. Let the initial polarization state in the experiment be chosen to be an arbitrary elliptically polarized state shown as the point $\mathrm{N}$ in figure 3. This could be achieved simply by orienting the first polarizer LP (fig. 1 of ref.[1]) suitably, without disturbing the quarterwave plate. Let $\theta$ be the polar angle of the point N. The circuits traced by the two beams are NPL and NQL, where NP, PL, NQ and QL are geodesic curves and the interferometer records the phase equal to half the solid angle of the circuit NPLQN, which is equal to the phase of the complex number $c=<N|P><P| L><L|Q><Q|$ $N>$. This changes nonlinearly in general as the polarizer LA is rotated.

Figure 4 shows, for different values of $\theta$, the computed phase shifts expected in such an experiment as a function of the orientation of the analyzer LA (fig. 1

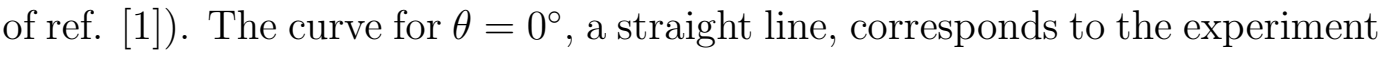
actually reported. The interesting feature is the $\pm 2 \pi$ jump near $\theta=90^{\circ}$ and $\phi=90^{\circ}$ (see curves $\mathrm{C}$ and $\mathrm{D}$ ). This is a new result and in fact contradicts the conclusion reported in ref. [19], namely in polarization interference experiments one sees only $\pm \pi$ phase jumps. The earlier conclusion holds in the context of experiments where only one of the scalar products forming the product $c$ goes to zero at a time. In the present experiment, at the singular point in the parameter space, two scalar products in the product go to zero simultaneously. This can double the strength of the singularity. 


\section{The BK Experiment}

In one of the experiments reported in ref. [2], Berry and Klein consider propagation of polarized light through a sequence of $\mathrm{N}$ polarizers that transmit states $\left|\mathbf{r}_{\mathbf{1}}>,\right| \mathbf{r}_{\mathbf{2}}>, \ldots, \mid \mathbf{r}_{\mathbf{N}}>$, represented respectively by points $\mathbf{r}_{\mathbf{1}}, \mathbf{r}_{\mathbf{2}}$, $\ldots, \mathbf{r}_{\mathbf{N}}$ on the Poincaré sphere which are distributed uniformly on a small circle with polar angle $\theta$. Under such a propagation, which is equivalent to $\mathrm{N}$ projections, the beam acquires a pure geometric phase which, for large $\mathrm{N}$, is equal to the solid angle subtended by the small circle at the centre of the sphere. The actual experiment is done for $\mathrm{N}=4$ and $\theta=90^{\circ}$, i.e. with 4 linear polarizers. The authors observe a fringe shift of magnitude $\pi$, as expected.

Let us note that the passage of a light beam through a polarizer is also accompanied with a large dynamical phase change of the U(1) type due to the refractive index of the polarizer material being different from 1 . In order to see only the phase shift due to the projections, the above dynamical phase must be reliably compensated with residual error small compared to the geometric phase being observed. What is observed in the present experiment is, therefore, not the phase difference but the change in phase difference between the two beams of the interferometer as the polarizers are rotated from a configuration when their easy axes are aligned to the final configuration. Presumably it is ensured beforehand that a rotation of the polarizers does not cause a change in the $\mathrm{U}(1)$ dynamical phase. What one observes, therefore, is a change in geometric phase as the circuit evolves with the rotation of the successive plates.

The point we wish to make is that if the experiment were performed with general polarizers corresponding to arbitrary values of $\theta$, it is possible in principle to keep track of the position of the fringes continuously as the polarizers are rotated one by one. The track of the polarization state at an intermediate 
stage when three of the polarizers have been rotated to their final configuration is schematically shown in figure 5 for two values of $\theta$, one in each hemisphere. Assuming the phase shift to be zero when all polarizers are aligned with the first one, the phase shift at the intermediate stage is given by half the solid angle subtended by one of the shaded areas in figure 5 . Note that the circuits in the upper and the lower hemisphere are traversed in the opposite sense, implying a phase shift of the opposite sign. The computed values of the phase shift as a function of the orientation angle $\phi / 2$ of the last polarizer rotated (i.e. the azimuth $\phi$ of the point D or D') for several values of $\theta$ are shown in figure 6 , where $\mathrm{N}=360$, a large number. The $\pm \pi$ phase jump near $\theta=90^{\circ}$ and $\phi=180^{\circ}$ (see curves $\mathrm{C}$ and $\mathrm{D}$ ) is thus in principle observable. In the context of unitary transformations, an earlier experiment [22] aimed at demonstrating the $4 \pi$ spinor symmetry principle using polarization states, deals with similar circuits. The switch in the sign of the phase change in going from the upper to the lower hemisphere has been observed in this experiment. Let us note that the standard result is that the geometric phase for a complete circuit of the above type is equal to $\pi(1-\cos \theta)$, irrespective of the value of $\theta$. This does not contain such a jump.

\section{Summary}

Our analysis of three experiments aimed at measuring a pure geometric phase as formulated by Pancharatnam reveals that in every case a singularity in the parameter space of the experiment where the phase becomes undefined is an essential feature of the problem. While in the IWBS expt. the singularity has been demonstrated in detail, in the HRSS and the BK expts. these are 
potentially measurable. In the context of unitary transformations, such singularities have been seen in earlier polarization experiments [23, 24]. While all the singularities observed so far have strength \pm 1 yielding a total phase change around a circuit equal to $\pm 2 \pi$, the singularity in the HRSS expt. has strength \pm 2 and would be interesting to observe.

\section{Note added :}

One of the referees has expressed the view (probably based on the conclusions of ref. 25]) that the principle of the IWBS experiment is the same as that of the Senarmont compensator used in interferometers in the sixties and the seventies. We disagree. The Senarmont compensator falls into the class of polarimetric devices that sense the polarization state of a beam with the help of retarders and polarizers. The IWBS experiment is an interferometric technique that senses the phase shift between two beams. The two important constraints of equal amplitudes in the two polarizations and a special orientation of the quarterwave plate that takes the two linear polarizations to the circularly polarized states in the Senarmont compensator are not present in the IWBS experiment. It is also not clear how nonlinear phases measured in the IWBS experiment would be measured with a Senarmont compensator. 


\section{References}

[1] Hariharan, P., Ramachandran, H., Suresh K.A. and Samuel, J., 1997, J.Mod.Opt. 44, 707.

[2] Berry, M.V. and Klein, S., 1996, J. Mod. Opt. 47165.

[3] Pancharatnam, S., Proc. Indian. Acad. Sci., 1956, A 44, 247.

[4] Bhandari, R., 1993, Phys. Lett. A, 18021.

[5] Berry, M. V., 1984, Proc. Roy. Soc. London A 392, 45.

[6] Collected works of S. Pancharatnam, 1975, Oxford University Press.

[7] Bhandari, R. and Samuel, J., 1988, Phys. Rev. Lett., 60, 1211. This experiment is sometimes incorrectly attributed in literature to Pancharatnam. While Pancharatnam's experiments demonstrated the presence of the geometric phase, these do not constitute a quantitative measurement of the geometric part of the phase.

[8] Bhandari, R., 1988, Phys. Lett. A, 133, 1.

[9] Simon, R., Kimble H. J., and Sudarshan, E. C. G., 1988, Phys. Rev. Lett., 61, 19.

[10] Chyba, T.H., Wang, L. J., Mandel, L. and Simon, R., 1988, Opt. Lett., 13, 562 .

[11] Tompkin, W.R., Malcuit, M.S., Boyd, R.W. and Chiao, R.Y., 1990, J. Opt. Soc. Am., B7, 230. 
[12] Kwiat P.G. and Chiao, R.Y., 1991, Phys. Rev. Lett., 66, 588.

[13] Berry, M.V., 1987, J. Mod. Opt., 34, 1401.

[14] Wagh, A.G., Rakhecha, V.C., Summhammer, J., Badurek G., Weinfurter, H., Allman, B.E., Kaiser, H., Hamacher, K., Jacobson, D.L. and Werner, S. A., 1997, Phys. Rev. Lett. 78, 755.

[15] Hariharan, P. and Roy, M., 1992, J. Mod. Opt., 39, 1811.

[16] Hariharan, P. and Roy, M., 1994, J. Mod. Opt., 41, 2197.

[17] Hariharan, P., 1995, seminar given at the Raman Research Institute.

[18] Bhandari, R., 1991, Phys. Lett. A, 157, 221.

[19] Bhandari, R., 1997, Phys. Rep., 281, 1.

[20] Schmitzer, H., Klein, S. and Dultz, W., 1993, Phys. Rev. Lett., 71,1530 .

[21] Bhandari, R., 1989, Phys. Lett. A, 135, 240.

[22] Bhandari, R., 1993, Phys. Lett. A, 180, 15.

[23] Bhandari, R., 1992, Phys. Lett. A, 171, 262.

[24] Bhandari, R., 1992, Phys. Lett. A, 171, 267.

[25] Hariharan, P., 1993, J. Mod. Opt. 40, 2061. 


\section{Figure Captions}

Figure 1: The QWP oriented at an angle $-45^{\circ}+\theta / 2$ to the $\mathrm{x}$-axis in real space rotates the initial states $\mid A>$ and $\mid \tilde{A}>$ to the states $\mid P>$ and $\mid \tilde{P}>$ about the point $\mathrm{Q}$ which represents the orientation of its fast axis. The linear polarizer brings the states $\mid P>$ and $\mid \tilde{P}>$ along the shorter geodesics to the state $\mid N>$ which rotates along the equator of the Poincaré sphere through $2 \tau$ as the polarizer is rotated through $\tau$. The interferometer measures a phase equal to half the solid angle subtended by the shaded area $P N F \tilde{P} L Q P$, bounded by the two geodesics.

Figure 2: The solid line in the curves shown in (a)-(h) represents the computed phase change as a function of $2 \tau$ for a few chosen values of the orientation of the QWP which is equal to $-45^{\circ}+\theta / 2$. The dots represent the measured values. The state $\mid N>$ rotates on the sphere through an angle $2 \tau$ when the polarizer rotates through $\tau$.

\section{Figure 3:}

The two beams in the interferometer follow the tracks shown as NPL and NQL on the Poincaré sphere. The measured phase difference is half the solid angle of the area NPLQN which changes nonlinearly as the linear polarizer LA rotates. The singularity occurs when the initial state $\mathrm{N}$ is linear and coincides with $\mathrm{A}$ and the angle $\phi$ equals $180^{\circ}$. The initial state $\mathrm{N}$ is varied by changing the orientation of the first polarizer in front of the laser.

Figure 4: The computed phase change as a function of the orientation $\phi / 2$ of the linear analyzer for various values of the polar angle $\theta$ of the initial state $\mathrm{N}$. Note the $\pm 2 \pi$ phase jump near the value $\theta=90^{\circ}$ and $\phi / 2=90^{\circ}$. 


\section{Figure 5:}

The polarization state follows the track ABCDFA (A'B'C'D'F'A') on the Poincaré sphere at an intermediate stage when the first polarizer corresponds to the state $A\left(A^{\prime}\right)$ and three of the polarizers have been rotated to their final orientations corresponding to the points B (B'), C (C') and D (D').

\section{Figure 6:}

The computed phase change as a function of the orientation $\phi / 2$ of the last rotated polarizer for various values of the polar angle $\theta$ of the circuit. Note the geometric phase for the full circuit flips from $+\pi$ to $-\pi$ near $\theta=90^{\circ}$. 
This figure "figure1.JPG" is available in "JPG" format from: http://arxiv.org/ps/physics/9810045v2 
This figure "figure2.JPG" is available in "JPG" format from: http://arxiv.org/ps/physics/9810045v2 


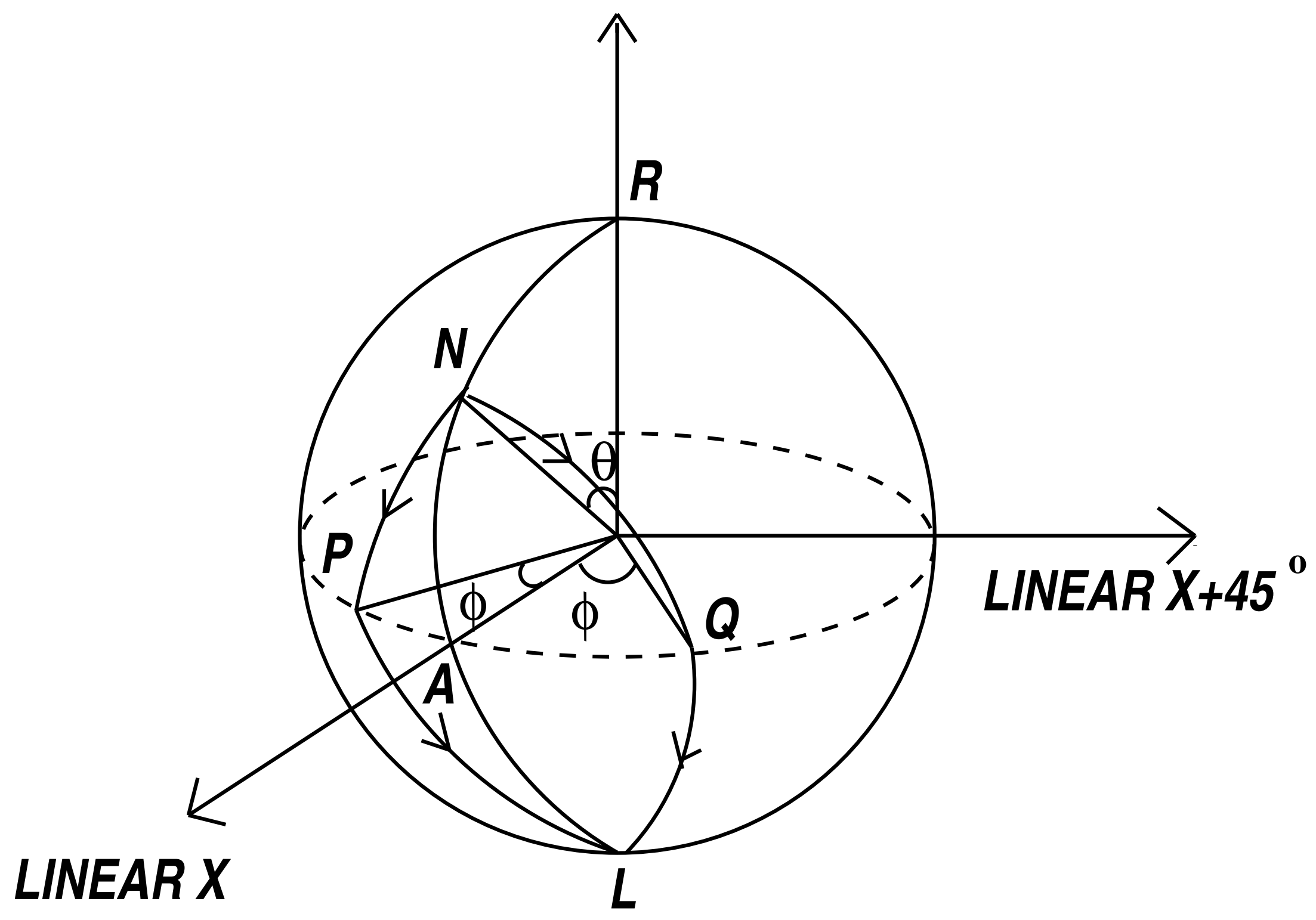




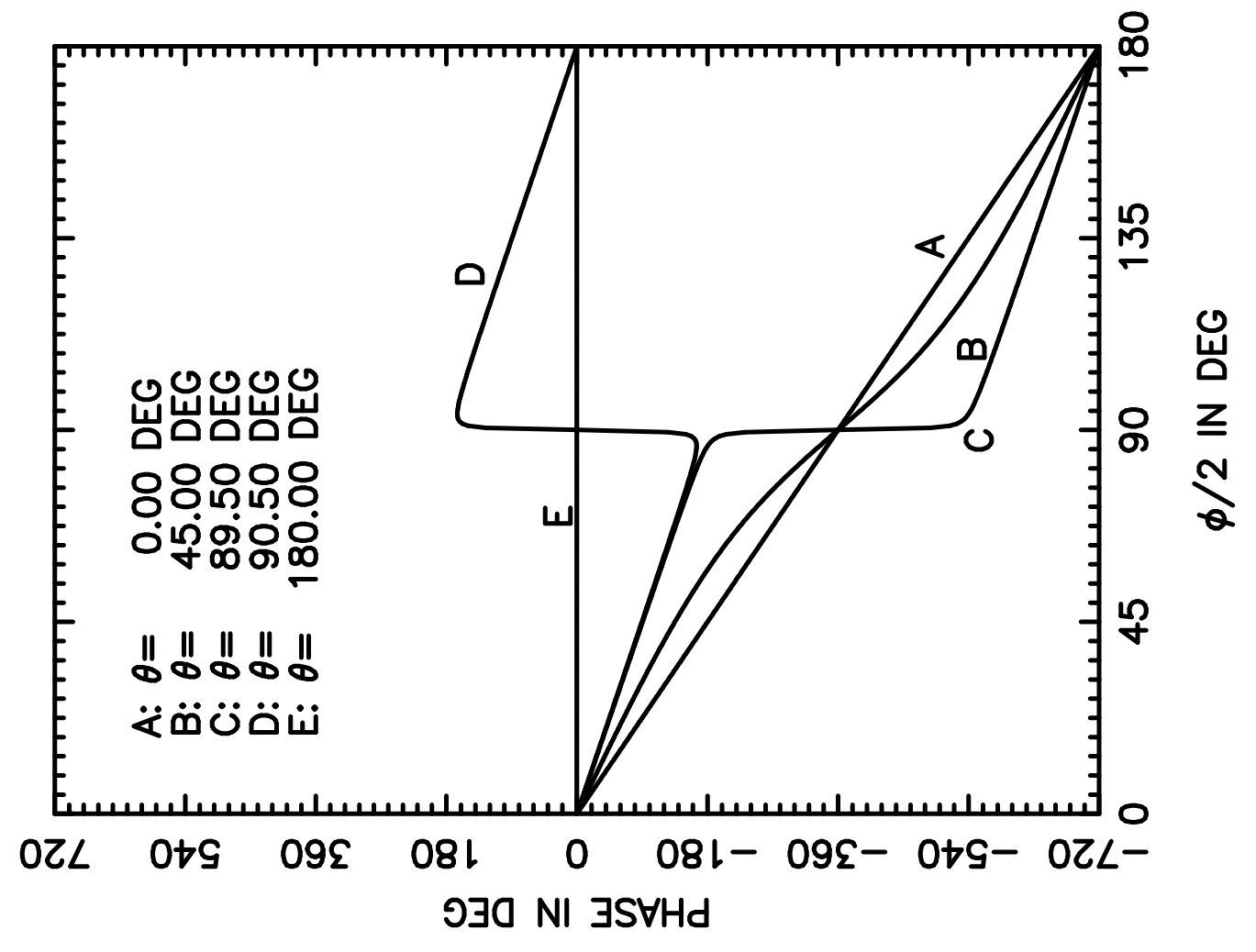




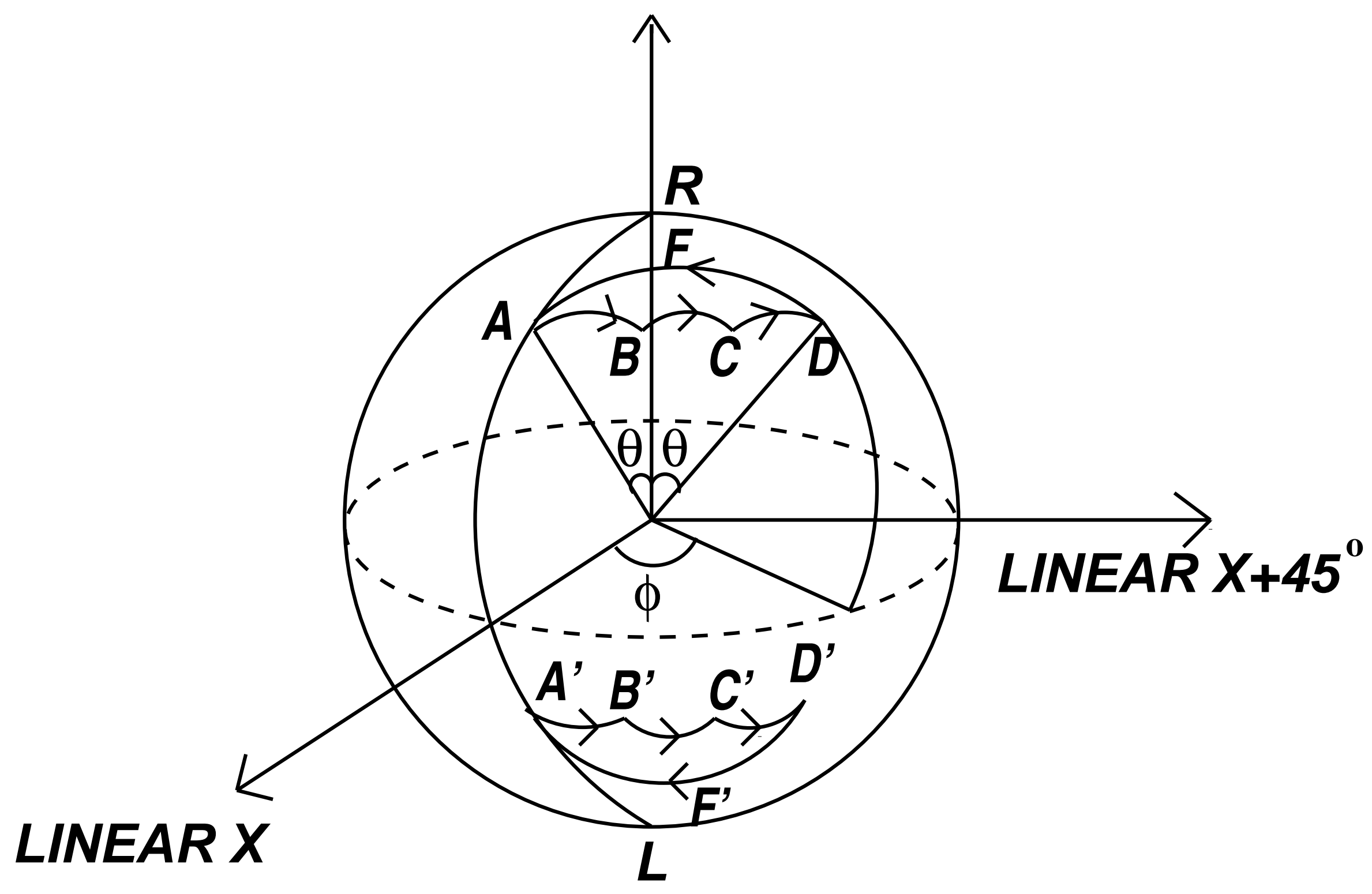




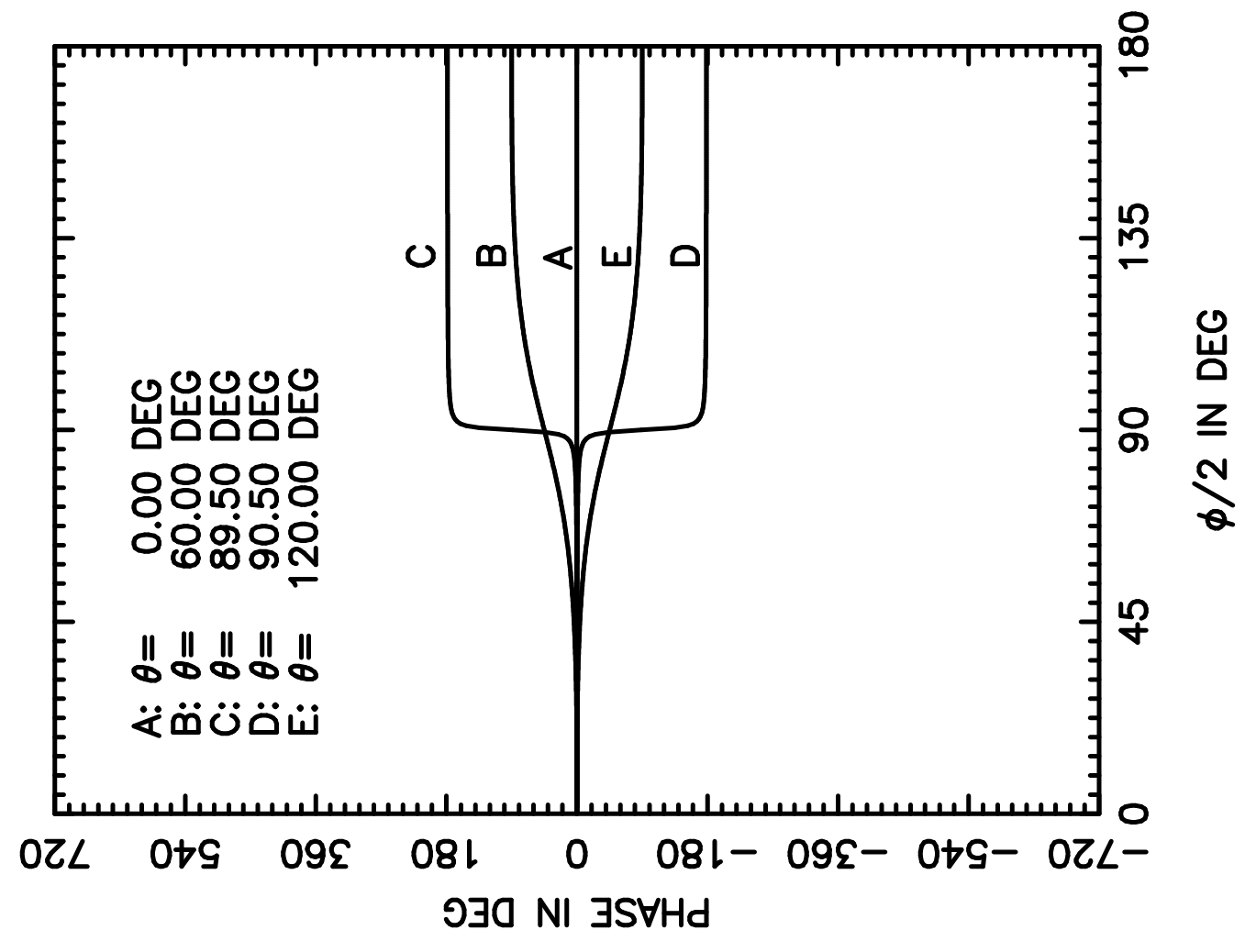

